\title{
¿EL JUEGO EN LA INFANCIA PUEDE HUIR DE LA HETERONORMATIVIDAD? ${ }^{1}$
}

A brincadeira na infância pode fugir da heteronormatividade?

May the childhood's games escape from the heteronormative?

\section{José Manuel Alvarez Seara}

ISEF-CURE-UDELAR Fono: 0059899428315. Correo electrónico: josmanu3@ gmail.com

\section{Resumen}

El presente texto es parte de mi trabajo de tesis de maestría en Educación Física de la Universidad Federal de Santa Catarina, Brasil (2015). En el mismo me propongo cuestionar si el juego puede huir de los determinismos de género. La pregunta provocativa del título, si se puede decir así, indaga si el juego en la infancia puede huir de una marcada heteronormatividad (Butler, 1999) que invade y se proclama como hegemónica en nuestros cuerpos. Reina y Cea (2005) atribuyen a los juegos ciertos estereotipos de género, en ese sentido Mc Phail Fanger (2006) va a decir que ciertos patrones se inculcan y reproducen en la infancia, resultado de esa reproducción es el que se le asigna a las niñas para jugar muñecas y a los varones autitos.

La investigación fue realizada en un barrio del oeste de Montevideo, Uruguay, en el que se entrevistaron a personas adultas, padres y madres de niñas/x/os menores de 10 años de edad. La metodología utilizada fue predominantemente cualitativa, estudio de caso, donde se realizaron observaciones, entrevistas en profundidad y donde luego se relacionaron las observaciones y lo que esas personas manifestaron con la teoría.

Se observó que las personas adultas mayoritariamente reproducen ciertos estereotipos de género en los juegos que realizan con sus hijxs, y son pocas las personas adultas que no reproducen patrones heteronormativos.

Este trabajo pretende aumentar las investigaciones que se vienen realizando en relación al juego y la infancia, desde una perspectiva de género y diversidad sexual.

\footnotetext{
${ }^{1}$ Este artículo es parte de mi trabajo de maestría financiada por la beca CAPES de Brasil, para docentes de la UDELAR, Uruguay.
} 
Palabras clave: juego, infancia, género, diversidad sexual, heteronormatividad.

\begin{abstract}
This text is part of my Master's thesis in Physical Education at the Federal University of Santa Catarina, Brazil (2015). In it I propose to question whether the game can escape gender determinism. The provocative question of the title, if you can say so, inquires whether the game in childhood can escape from a heteronormativity (Butler, 1999) that invades and proclaims itself as hegemonic in our bodies. Reina and Cea (2005) attribute certain gender stereotypes to games. On the other hand, Mc Phail Fanger (2006) goes on to say that certain patterns are inculcated and reproduced in childhood, the result of which reproduction is assigned to them the girls to play dolls and the boys cars.
\end{abstract}

The research was done in a neighborhood in western Montevideo, Uruguay, where adults, fathers and mothers of girls/boys/xs under 10 years of age were interviewed. The methodology used was predominantly qualitative, a case study, where observations were made, in-depth interviews and where the observations were later related and what those people said with the theory.

It was observed that adults mostly reproduce certain gender stereotypes in the games they play with their children, and few adults do not reproduce heteronormative patterns.

This work aims to increase the research that is being done in relation to games and childhood, from a gender and sexual diversity perspective.

Keywords: games, childhood, gender, sexual diversity, heteronormativity.

\title{
Resumo
}

Este texto faz parte da minha tese de Mestrado em Educação Física na Universidade Federal de Santa Catarina, Brasil (2015). Por meio deste, proponho questionar se a brincadeira pode escapar do determinismo de gênero. A pergunta provocativa do título questiona se a brincadeira na infância pode fugir de uma marcada heteronormatividade (Butler, 1999) que invade e se proclama como hegemônica em nossos corpos. Reina e Cea (2005) atribuem às brincadeiras certos estereótipos de gênero. Por outro lado, Mc Phail Fanger (2006) diz que certos padrões são introduzidos e reproduzidos na infância, de modo que cabe às meninas brincar de bonecas e aos meninos, com carrinhos. 
A pesquisa foi realizada em um bairro no oeste de Montevidéu, Uruguai, onde foram entrevistados adultos, pais e mães de meninas/os/xs com menos de 10 anos de idade. A metodologia utilizada foi predominantemente qualitativa, estudo de caso, onde foram feitas observações e entrevistas em profundidade, as quais foram posteriormente relacionadas à teoria.

Observou-se que os adultos reproduzem certos estereótipos de gênero nas brincadeiras que eles realizam com seus filhos/as, sendo que poucos adultos não reproduzem padrões heteronormativos.

Este trabalho tem como objetivo incrementar a pesquisa que está sendo feita em relação às brincadeiras e à infância, sob uma perspectiva de gênero e diversidade sexual.

Palavras chave: brincadeira, infância, gênero, diversidade sexual, heteronormatividade.

\section{Introducción}

El presente trabajo es parte de mi tesis de maestría de la UFSC ${ }^{2}$ que termine en el año 2015, donde mi tutor fue el Dr. Jaison José Bassani de la PPGEF³-UFSC, mi co-tutora la Dra. Karina Batthyány de la FCS-UDELAR ${ }^{4}$, y contó con el apoyo y financiación para docentes de la UDELAR de la $\mathrm{CAPES}^{5}$, Brasil.

La investigación se desarrolló en un barrio del oeste de Montevideo, Uruguay, donde realice observaciones en los espacios públicos de dicho barrio y entrevistas a personas adultas a cargo de niñas/os menores de 10 años de edad. Fue un estudio predominantemente cualitativo, estudio de caso (Coller, 2000) en el que se observaron que juegos hacían las personas adultas con lxs/os/as niñ/xs/os/as y se les preguntó a las personas adultas a qué juegos jugaban con sus hijos/as.

Se entrevistaron un total de 15 personas adultas, que estaban a cargo de los cuidados de niños/as/xs menores de 10 años de edad.

El presente trabajo reflexiona sobre los juegos que las personas adultas realizan con los/as niñas/os en los hogares y en los espacios públicos. La investigación tiene una mirada de género y

\footnotetext{
${ }^{2}$ Universidade Federal de Santa Catarina, Santa Catarina, Brasil.

${ }^{3}$ PPGEF programa de posgraduación en educación física de la UFSC.

${ }^{4}$ UDELAR Universidad de la República, Uruguay.

${ }^{5}$ CAPES Coordinadora de perfeccionamiento de enseñanza superior, Brasil.
} 
diversidad sexual, que pretende ser provocativa, cuestionando una de las máximas sobre que el juego es algo muy bueno para la infancia.

En la investigación llevada a cabo, aunque no se puede generalizar porque es un estudio de caso, se puede evidenciar que los juegos infantiles que realizan las personas adultas entrevistadas y observadas con las/os/xs niñas/os/xs pueden dejar marcas en los cuerpos de esas/o/xs niños/a/xs, esas marcas son producto de una reproducción de ciertos estereotipos de ser hombre y de ser mujer, no dejando lugar, en la mayoría de los casos, para la experimentación de otras formas de ser que no sean heteronormativas (Butler, 1999).

Este trabajo pretende cuestionar el papel asignado al juego en la infancia, debatiendo si estos no fijan ciertos estereotipos de género. El presente trabajo pretende aportar y aumentar el cuestionamiento que viene siendo realizado por diversos/a/xs autoras/es/x a la heteronormatividad (Butler, 1999; Preciado, 2002; Braidotti, 2000), haciendo foco en la pregunta si los juegos infantiles pueden ser usados por las personas adultas para reproducir y sedimentar ciertos patrones heteronormativos en los/as/xs niñxs/as/os.

\section{Metodología de investigación}

El trabajo indaga sobre los discursos y la perspectiva que las personas adultas tienen de los juegos que realizan con niñas/o/xs en el tiempo de cuidados infantiles. Se entrevistaron personas adultas responsables por los cuidados infantiles, de un barrio de Montevideo, residentes en un grupo de casas en contexto urbano, y que tenían a su cargo niños/a/xs de hasta 10 años de edad. Se delimitó esa fase etárea en función del "ciclo de vida de expansión", caracterizado por la edad de las niñas/o/xs menores ( 0 a 10 años), que imponen a los adultos mayores restricciones de tiempo y mayor disponibilidad y energía para el cuidado de niñas/os.

Objetivando un mayor detalle y diversidad de los sujetos entrevistados, se dividió la muestra en 3 grupos, en función de la edad de los/as niños/as: de 0 a 2, de 3 a 5 y de 6 a 10 años.

Para demarcar la temporalidad social. Las visitas a las residencias donde viven los/as entrevistados/as fueron realizadas en días diferentes, tanto durante la semana (día de trabajo) como en el fin de semana (día de no trabajo). Las entrevistas fueron realizadas entre los meses de agosto a octubre de 2014, y se llegó al punto de saturación cuando fueron realizadas 15 entrevistas en 
profundidad. El mencionado punto de saturación fue identificado por la repetición de las respuestas por parte de las personas entrevistadas en relación a las dimensiones y objetivos del trabajo. Se debe tener en cuenta que el presente trabajo, al caracterizarse como un estudio de caso, no pretende generalizar sus conclusiones.

La metodología utilizada en la investigación fue el estudio de caso, que según Coller (2000) se caracteriza por tener como objeto un fenómeno contemporáneo, en un contexto real y cotidiano, donde el fenómeno no puede ser manipulado. Se realizaron entrevistas en profundidad, que sirvieron para la reconstrucción de acciones pasadas, el estudio de las representaciones sociales (estereotipos, normas) y las interacciones que se observan entre las/o/xs actoras/e/xs sociales (Delgado y Gutierrez, 1999). Así como se realizaron observaciones en espacios públicos, en la que se ejecuta una tarea de investigación desde "dentro" de las realidades humanas que pretende abordar. La observación es el principal instrumento de trabajo de la etnografía, por ejemplo, y apoyo para generar las "impresiones" del investigador que son "registradas" en el llamado diario de campo. Se trata de un registro continuo y acumulativo de todo lo que sucede y es captado por el/la investigador/a (Corbetta, 2007).

\section{Los juegos infantiles desde una perspectiva de género}

Walter Benjamin (1928) va a escribir sobre el juego, el juguete y su historia cultural, así como sobre la infancia, para el autor el juego es algo que los niños/a/xs repiten hasta el cansancio, es el otra vez. Asimismo los juguetes son objetos que no tienen nada que ver con la infancia, sino con las personas adultas, pero la óptica infantil mira esos objetos y les da otro sentido. Los juguetes para el autor están vinculados al mundo de las personas adultas.

Reina y Cea (2005) en su investigación sobre juegos y género, identifican otras investigaciones ya realizadas sobre las relaciones de género e infancia, que confirman que niños/a/xs demuestran comportamientos, preferencias, competencias, atributos de personalidad más apropiados para su sexo biológico, siguiendo, desde temprana edad, normas y patrones de género establecidos social y culturalmente.

En este sentido también los autores Caldas-Coulthard y Van Leeuwen (2004) plantean como los juguetes (así como los textos e imágenes que acompañan esos objetos materiales) son 
desde una perspectiva semiótica significativos en lo que se refiere a las relaciones de género en la infancia, e indagan cómo algunos de sus significados son producidos. Estos autores dicen que los juguetes son un "microcosmos del mundo adulto" y siempre significan alguna cosa:

esta alguma coisa é sempre inteiramente socializada, constituída pelos mitos ou pelas técnicas da vida adulta moderna. Brinquedos representam, basicamente, as instituições de nossas sociedades: o Exército, a Radiodifusão, os Correios, a Medicina (maletas de médico em miniatura, salas de cirurgia para bonecas), a Escola, o Salão da Cabeleireira (secadores para fazer permanente), o Transporte (trenes, Citroens, Vedettes, Vespas, postos de gasolina) e a Ciência (Brinquedos espaciais, ferramentas, etc.) (CaldasCoulthard y Van Leeuwen, 2004: 13-14).

Ariés (1987) manifiesta, que el juego es uno de los elementos más importantes a tener en cuenta como un indicador social para visualizar la categoría generacional de infancia.

Asimismo para Fernández (1994), la infancia no es un dato, pero sí una producción histórica y social. En este sentido, la autora afirma que no siempre hubo "la niña", y que no todas las niñas son parte de una sociedad con prácticas semejantes de infancia, ni responden a un mundo de significados comunes.

Agamben (2012), por otro lado, va a manifestar en Teología y Lenguaje, en el capítulo Por una filosofía de la infancia, que la infancia es la preeminente composición de lo posible y de la potencia. Lo que caracteriza a la infancia es su propia potencia, dirá el autor. La infancia vive su propia posibilidad (Agamben, 2012: 29). En ese mismo texto, el filósofo italiano dirá que es en vano que las personas adultas intenten confinar a las/o/xs niñas/o/xs a tiempos y lugares limitados, como son los jardines de infantes o centros infantiles, a juegos codificados, el tiempo de jugar y los cuentos de hadas, por ejemplo. Para Agamben

“los niños saben que la cuestión no es fantasear, sino que en ese experimento los/a/xs niños/a/xs se juegan toda su vida, estando en juego a cada instante“ (Agamben, 2012: 30).

Agamben (2007), en su libro Infancia e historia, dedicará un capítulo a los juegos y juguetes. En esa sección de su obra, que dedica a Levi Strauss, va a afirmar que las esferas del juego y de lo sagrado están estrechamente ligadas, y que muchos de los juegos que conocemos hoy 
se encuentran en antiguas ceremonias sagradas, en danzas, luchas, rituales y prácticas adivinatorias: “.Así en el juego de la pelota podemos discernir las huellas de la representación ritual de un mito en el cual los dioses luchaban por la posesión del sol; la ronda era un antiguo rito matrimonial; los juegos de azar derivan de prácticas oraculares; el trompo y el damero eran instrumentos adivinatorios“" (Agamben, 2007: 99).

Para Agamben (2007), el juego viene de lo sagrado, por ende, el juego también puede modificar lo sagrado. Para el autor, el juego tiende a destruir la conexión entre pasado y presente, y sí el rito es entonces una máquina para transformar la diacronía en sincronía, el juego es, por lo contrário, una máquina que transforma la sincronía en diacronía:

o jogo libera e desvia a humanidade da esfera do sagrado, mas sem a abolir simplesmente. O uso a que o sagrado é devolvido é um uso especial, que não coincide com o consumo utilitarista. Assim, a "profanação" do jogo não tem a ver apenas com a esfera religiosa. As crianças, que brincam com qualquer bugiganga que lhes caia nas mãos, transformam em brinquedo também o que pertence à esfera da economia, da guerra, do direito e das outras atividades que estamos acostumados a considerar sérias (Agamben, 2007: 61).

Coincido con lo que dice Agamben (2007) de que el juego tiene ese potencial transformador (Agamben, 2007).

Asimismo Scheines (1999) va a decir que el juego es un puente de relacionamiento con el mundo y que rompe con la realidad de todos los días:

Jugar es interrumpir el orden que rige la vida cotidiana, romper ese mapa que nos sirve para manejarnos en la realidad de todos los días, y sumergirnos en la realidad colmada de objetos tal como aparecen. Por eso cito la famosa frase de Oscar Wilde: el mundo del jugador es pura apariencia donde los detalles son tan importantes como los rasgos sobresalientes. El que juega, juega con detalles, no desdeña la complejidad de las cosas. Entonces, jugar es interrumpir el orden de la vida cotidiana y habitar la plenitud caótica, abigarrada, llena, donde puedo manipular de la manera que quiera (Scheines, 1999: 4).

Continuando con el artículo, en el ámbito de esta investigación surge un tensionamiento entre los estereotipos de género asociados a los juegos, los juguetes y la infancia. Se podría 
preguntar si es posible huir o sobrepasar estos estereotipos, patrones heteronormativos o regulatorios observados en los juegos infantiles y los juguetes (Butler, 1999).

Butler (2002) va decir que la categoría sexo es un principio normativo, que significa para la autora el mismo que Foucault llamó de ideal regulatorio en sus trabajos. Para Butler el sexo no sólo funciona como norma, sino que también es una práctica reguladora que produce los cuerpos que gobierna, cuya fuerza se manifiesta como una especie de poder productivo, capaz de demarcar, circunscribir y diferenciar los cuerpos que controla.

En otras palabras la autora (Butler, 2002) expresa la idea de que el género y la sexualidad, así como el propio sujeto, no preexiste al uso que hacemos del lenguaje en nuestras prácticas discursivas cotidianas. No somos nosotros, en ese sentido, que hacemos algo con el lenguaje, es el lenguaje que hace algo con nosotros, nos constituye. En este sentido el género es siempre un hacer como dice Butler (1999). Asimismo la categoría género es una categoría en debate, Scott (2008) manifiesta que el uso de la conceptualización de género pone de relieve un sistema completo de relaciones que puede incluir el sexo, pero no está directamente determinado por el sexo, o es directamente determinante de la sexualidad. Scott (2008) va a insistir en que la categoría "género" es útil solo como pregunta, manifestando que las preguntas sobre el género nunca quedan completamente respondidas.

El sexo refiere a las características y diferencias genéticas, hormonales y anatómicas que distinguen a las personas en machos, hembras e intersexuales. Esta definición se encuentra actualmente en discusión, ya que han surgido corrientes de pensamiento que también conciben al sexo como una construcción social (Butler, 1999).

Butler (1999) va a decir que existe una matriz cultural que instituye la heterosexualidad normativa como norma y que todo desvío que no coincide con esa norma es juzgado. En ese sentido, determinados tipos de identidad no puede existir, porque no se adecuan al patrón heteronormativo, en el cual el género deriva del sexo anatómico (Butler, 2002).

Tal vez sea legítimo pensar el juego como un dispositivo que impone significaciones como legítimas ligadas a una heteronormatividad (Butler, 1999) en forma de estereótipos de género, que 
son invisibles para muchos, y que, como son vistas como legítimas, se tornan muy difíciles de ser contrariadas.

El juego, se transforma así en una de sus facetas, en un posible transmisor de patrones de comportamientos a seguir o rechazar, en este sentido los padres, madres, personas adultas, otros miembros de la familia y cuidadores son los encargados, por lo que se observa en esta investigación, entre otros de llevar a cabo dicha transmisión en el hogar. En este trabajo procuré indagar sobre las percepciones que tienen las personas adultas en relación a los juegos infantiles, que juegos realizan con los/as niñas/os, quienes los realizan, en qué espacios del hogar y espacios públicos, y cuáles son los juegos prohibidos que no pueden jugar los/as/xs niños/as/xs.

El juego es también una forma de los/a/xs niñas/o/xs de interactuar en/con el mundo, que produce la satisfacción por medio de sensaciones, como de nuevas experiencias, que permiten percibir y expresar, aprender e interactuar. En este sentido Berger y Luckmann (1986) analizan y clasifican patrones de transmisión del mundo social que ocurren a través de la socialización, por medio de la internalización y reconocimiento de procesos sociales. Este tipo de socialización, de producirse como miembro de una sociedad, en el caso de las/o/xs niñas/o/xs, es dirigido principalmente por las madres, padres, personas adultas, otros miembros de la familia, cuidadores y profesore/a/xs. En este proceso está implicada la identificación de valores, preconceptos, ideales, sentimientos y proyecciones de esas personas significativas para las/o/xs niñas/o/xs: “Te podes imaginar que dos nenas con un varón, y ellas son nenas, juegan a las muñecas, las peinan, las cambian, o juegan a que hacen comida y cosas así, él es el papá y ellas son las hijas, cosas asî́ (Carolina, 35 años, 2 hijas y 1 hijo).

Así como se puede considerar que Carolina estuviese apenas "describiendo" el juego de sus hijas con el hermano, y de que el niño/x asume "normalmente", como sería de esperar, el papel que correspondería a un niño/x en un juego de roles (de "padre"), el hecho es que hay aquí una reproducción de expectativas y funciones sociales históricas y culturalmente atribuidas a hombres y mujeres (Scott, 1986). Haciendo una analogía con lo que expresan Berger y Luckman (1986) cuando hablan de socialización, se podría cuestionar en qué medida es el juego un agente de socialización privilegiado de identidades y valores. 
Reina y Cea (2005) afirman que los estereotipos de género son patrones de comportamiento aprendidos a través de la experiencia, en el cual niñas/o/xs tradicionalmente experimentan y adquieren ciertos comportamientos asociados a patrones "masculinos" y/o "femeninos", por medio de los juegos. Reciben lo/x/as niñas/x/os la apropiación social al hacer actividades propias de su sexo biológico, y son corregidos en sus preferencias cuando estas no corresponden a los estereotipos tradicionales. Por lo tanto, los/x/as niñas/x/os progresivamente internalizan patrones adecuados para su comportamiento de género, para poder actuar en conformidad con estos patrones. Los autores afirman que es en ese momento que se produce una "conciencia de género", al diferenciarse juegos para niñas y otros para niños (Reina y Cea, 2005).

Corroborando con lo que manifiestan Reina y Cea (2005), las personas adultas entrevistadas para esta investigación relatan en sus discursos mayoritariamente, que juegan con los niño/xs a juegos que tienen una relación con un universo "masculino", y juegan con niña/xs a juegos que hacen referencia a un universo "femenino", pero también se observan disidencias con esos universos: “yo soy más de hacerles, yo que sé, una casita y que ellas se metan y jueguen, claro, en el cuarto, yo les hago una casita más bien y ellos se quedan ahí quietitos y se quedan jugando, y él (compañero) es más varonil, juega a la pelota con la hija“ (Milena, 21 años, 2 hijas y 1 hijo).

Si, acá se juega todo el día. Juego de manos con ellos, si juego de manos, para que aprendan a hacerse respetar, y todo, y que aprendan a defenderse también, ya de chiquito. Acá no hay varones, hay nenas que lo cagan a palos, y se tiene que hacer respetar ... Lucha, el chiquito no está, recién ahora está empezando a jugar, porque es maricón, muy maricón es, ponele como ahora yo estoy dibujando y viene y me garronea, me encaja un piñazo [golpe] y ahí empezamos, y empezamos a los piñazos suaves, este yo le pego para que le duela para que sienta y para que él pegue más fuerte, y así, no a lo bruto pero, hay un límite de golpe y de todo, te da y mira que te pega y te pega fuerte, pero tampoco porque me pegue una piña fuerte no le voy a pegar una piña más fuerte para que la sienta, le pego jugando, y ahora el chiquito se está adaptando a eso, yo le empiezo a pegar así y él empieza y me viene a pegar (Marcelo 33 años, 1 hijo y 1 hija).

Marcelo también nos dice en la secuencia de la entrevista que jugaba a la lucha también con su hija, "si, con ella también, estuve haciendo boxeo, estuve un año haciendo boxeo con ella" 
(Marcelo 33 años, 1 hijo y 1 hija), pero hace la aclaración de que "fue solo un año", porque ahora la niña no vive más con él, vive con su madre en otro lugar.

Aquí, en el último relato, se puede ver una disonancia con ciertos estereotipos de género, en la medida en que Marcelo juega a la lucha con su hija, un juego mucho más vinculado a un universo "masculino". En el mismo sentido, en la frase "acá no hay varones, hay nenas que lo cagan a palos" se puede observar una resistencia a ciertos estereotipos de género que podría estar también relacionada con la condición o estrato social, en la medida en que, en las clases populares, saberse defender (por medio de la lucha, por ejemplo) no es patrimonio solo de los niño/xs, es también patrimonio de las niña/xs. También debemos decir que después al entrevistar separadamente a la compañera actual de Marcelo, con quien tiene un hijo, ella nos dice al contrario de lo que relata Marcelo, que no le gustaba "jugar a la lucha” (juego de manos) cuando era niña/x, y que no le gusta que sus hijos/xs jueguen a eso, lo que sería un juego prohibido para ella.

conmigo no juegan a las peleas porque yo estoy en contra de que ellos jueguen de manos. Me entendés, porque ellos son chicos, por ahí uno grande se le va la mano porque no medís la fuerza con un niño de 5 años y por ahí le pegas mal y lo terminas lastimando, pero sin querer. No, entonces digo, yo estoy en contra de que jueguen de manos (En este caso, por motivos éticos, voy a proteger la identidad de la persona).

Es interesante percibir que la divergencia entre las dos personas adultas no está en la transmisión de determinados valores asociados al "juego de lucha” y a la representación de aquello que sería típicamente "masculino", como coraje, fuerza, soportar el dolor, saber defenderse etc. La divergencia que manifiesta ella en relación al compañero reside más en el hecho de, que para ella, los juegos como simulación de luchas pueden ser peligrosos y violentos porque, al no saber controlar la fuerza, los/as/xs niñas/os/xs pueden acabar, sin querer, lastimándose. Para Marcelo, por otro lado, saber pegar y soportar el dolor de un golpe todavía más fuerte del que aquel que él/la/x niña/o/x empleo tiene un papel educativo importante, inclusive para la hija/x. A partir de este ejemplo, notamos una diferencia interesante en el modo en como ambos interpretan aquello que sería adecuado en términos de cuidado y de juegos con los/a/xs hijos/a/xs. 
También Juan, padre de tres hijo/x/as (un niño/x de 2 años, y dos niñn/x/as, de 5 y 9 años de edad) nos relata lo que sus hijos/a/xs son incentivados/a/xs a/o les gusta jugar, donde se pueden evidenciar en el relato ciertos estereotipos de género en el juego y la elección de los juguetes:

Por lo general para el varón son autos, autitos, porque es fanático de los autos y la marca es Hot Wheels, vivimos comprando esa marca porque son los favoritos de él, y después las nenas varía un poco más. Yo que sé, hay más variedad de juguetes para las nenas que para el varón, pero por ejemplo muñecas, no compramos por ejemplo marcas de muñecas, tipo Barbie o esas cosas no, pero claro las muñecas siempre hay algún jueguito de té como hay, o máquinas de coser, como juegos recreativos también muchos (Juan, 30 años, 2 hijas y 1 hijo).

En relación al relato de Juan voy a recurrir a la autora Mc Phail Fanger (2006), que, parafraseando a Bourdieu, dirá que el conocimiento que las personas tienen de la lógica cotidiana las ayuda a abordar y resolver sus actividades diarias y el habitus, como patrón generador de disposiciones, rutinas y costumbres, se inculca y se adquiere desde la infancia y persiste a lo largo de la vida. Con base en el habitus, se producen (y reproducen) prácticas y percepciones que se ajustan a las restricciones del mundo social en el cual emergen, se consolidan y afianzan una cierta sensibilidad. Ese habitus se reproduce en que el juego con los autitos es entonces para los varones y el de las muñecas, juego de té para las nenas, como manifiesta Juan.

Conforme se ha expresado hasta el momento en el trabajo, y corroborando lo que Reina y Cea (2005) afirman sobre los estereotipos de género, existe en la mayor parte de los relatos de las personas entrevistadas, en relación a los juegos que hacen con sus hijos/a/xs, una transmisión de ciertos "patrones" y "valores" de género heteronormativos que refuerzan estereotipos y roles de género heteronormativos (Butler, 2002).

Asimismo Reina y Cea (2005) expresan que, al diferenciar los juegos, las/x/os niñas/x/os toman como referencia los modelos de su entorno relacionados con su sexo biológico: madres, padres, hermanas, hermanos, e inclusive personajes televisivos. Con base en estos autores, también es posible percibir en el relato de Juan mencionado anteriormente, como los juguetes elegidos por las personas adultas para lo/x/as niñas/x/os prevalecen ciertos estereotipos heteronormativos tradicionales que son transmitidos a las nuevas generaciones. Se puede decir que, desde esta 
perspectiva, ciertos estereotipos heteronormativos tradicionales son transferidos social y culturalmente a las nuevas generaciones por medio del juego y los juguetes (Reina y Cea, 2005).

Conforme se ha visualizado hasta aquí, el juego es una importante herramienta en la transmisión de valores sociales, así como de papeles relacionados con el género. Lo que se observa por ejemplo en los relatos de Juan, plantean la interrogante de qué pasaría si sus hija/xs prefiriesen los "autitos" a las muñecas. Evidentemente que la explicación para eso no está en el determinismo biológico, o en el determinismo social, está en los papeles sociales que históricamente atribuimos a los hombres y mujeres en nuestras sociedades, y en la heteronormatividad (Butler 1999).

En el trabajo de investigación realizado por Reina y Cea (2005), anteriormente referenciado, también se busca establecer, para el contexto de España, una posible relación entre los juegos y juguetes con el futuro personal, laboral y social de las/x/os niñas/x/os, según el género. Los autores citan como ejemplo las actividades que están relacionadas, tradicional y culturalmente, al género masculino, como la construcción, la mecánica, con juegos de estrategia y las que incorporan a las nuevas tecnologías, en las cuales existe según su investigación, una baja participación de las niñas. Esos juegos, para los autores, se transforman luego en una realidad social. En España, así como en Uruguay, profesiones como la de ingeniería son principalmente ocupadas por hombres, y las mujeres tienen mayor tendencia a elegir profesiones humanísticas (Reina y Cea, 2005).

\section{4. ¿Puede el juego no ser heteronormativo?}

Se debe tener en cuenta desde un primer momento, como ya mencionamos anteriormente, que este trabajo de investigación no se puede generalizar, porque es un estudio de caso y la muestra es muy pequeña (15 personas adultas entrevistadas).

En un primer momento, después de ver y analizar los relatos de las personas entrevistadas en esta investigación, podríamos decir que el juego en la mayoría de los casos no puede dejar de ser heteronormativo. Una de conclusiones que se desprende y se observa de este trabajo de investigación es que el juego no es algo neutro, por lo contrario, se evidencia en esta investigación que el juego puede dejar marcas indelebles (difíciles de borrar) en los cuerpos de las/o/xs 
niñas/o/xs, fruto de una cultura fuertemente marcada por una heteronormatividad, como manifiesta Butler (1999).

Se evidencian de las entrevistas y observaciones realizadas que predominan ciertos juegos para niña/xs (muñecas, juegos de té, juegos de roles) y ciertos juegos para niñx/os (autitos, lucha, pelota), así como ciertas prohibiciones de juegos según el género, los juegos que realizan las personas adultas entrevistadas reproducen mayoritariamente ciertos estereotipos de género determinados, que se relacionan con un cierto tipo de "deber ser "mujer y hombre heteronormativos (Butler, 1999).

Las personas adultas entrevistadas afirman en sus discursos mayoritariamente, que juegan con los niñas a juegos (y utilizan juguetes) que tienen relación con un cierto universo "masculino", y juegan con niñas a juegos (y utilizan juguetes) que hacen referencia un cierto universo "femenino".

Los niños son incentivados a jugar con pelotas, autos de colección, videojuegos, a jugar a ser papá en los juegos de roles y las niñas son incentivadas a jugar con muñecas, a las casitas, a hacer las labores de la casa (trabajo doméstico), a cocinar, a coser, a jugar a ser mamá en los juegos de roles.

Asimismo se puede observar de las entrevistas y las observaciones en espacios públicos coincidiendo con Bourdieu (1999), que predomina una violencia simbólica por parte de las personas adultas hacia las/os/xs niñas/os/xs. Las personas adultas imponen, avalan y prohíben juegos y juguetes según el sexo biológico de los/x/as niños/x/as, manteniendo un patrón heteronormativo (Butler, 1999).

Los juegos y juguetes son utilizados mayoritariamente por las personas adultas como herramienta y transmisores de ciertos valores sociales, así como de ciertos patrones y estereotipos de género, que se relacionan con papeles sociales que históricamente fueron atribuidos a hombres y mujeres (Scott, 1986). En este sentido son pocas las personas entrevistadas que se alejan de reproducir ciertos estereotipos de género.

Agamben (2007) ofrece una posible fisura en los juegos que brindaría la posibilidad de huir de esta heteronormatividad. Según el filósofo italiano, que plantea la necesidad de restituir el juego 
a su vocación puramente profana, como una tarea política (Agamben, 2007: 60). En esa profanación, los/as/xs protagonistas son las/os/xs niñas/os/xs, el juego podría cambiar esa heteronormatividad que atribuyen las personas adultas, ya que les niñ/a/o/xs podrían restituir su vocación profana al juego. Entonces el juego podría ser no heteronormativo.

Si consideramos como manifiesta Agamben (2007) que el juego tiene un potencial de transformación, y de que los juegos podrían cuestionar los estereotipos de género, el juego se puede transformar en un medio que puede cuestionar ciertos estereotipos de deber ser "hombre" y "mujer" (atribuidos a las personas en relación a su sexo biológico y en el marco de una heteronormatividad dominante) y promover una diversidad de formas de vivir y ser.

\section{Referencias bibliográficas}

Agamben, G. (2007) Infancia e historia. Destrucción de la experiencia y origen de la historia. Buenos Aires: Adrián Hidalgo Editora.

Agamben, G. (2007) Profanações. São Paulo: Boitempo Editorial.

Agamben, G. (2012) Teología y Lenguaje. Del poder de Dios al juego de los niños. Buenos Aires: Las cuarenta.

Alvarez Seara, J.M. (2015) Cuidados infantis, brincadeiras e gênero: estudo em um bairro de Montevidéu, Uruguai. Florianópolis: UFSC.

Aries, P. (1987) El niño y la vida familiar en el antiguo régimen. España: Taurus.

Benjamin, W. (1928) Historia cultural del juguete. En Giulio Schiavoni, Escritos. La literatura infantil, los niños y los jóvenes. Buenos Aires: Nueva Visión, 1989.

Benjamin, W.(1928) Juguete y juego. En Giulio Schiavoni, Escritos. La literatura infantil, los niños y los jóvenes. Buenos Aires: Nueva Visión, 1989.

Berger, P. y Luckmann, T. (1986) La construcción social de la realidad. Buenos Aires: Amorrortu. Bourdieu, P. (1999) Razones Prácticas. Sobre la teoría de la acción. Barcelona: Anagrama.

Braidotti, R. (2000) sujetos nómades. Corporización y diferencia sexual en la teoría feminista contemporánea. Buenos Aires, Paidós. 
Butler, J. (1999) El género en disputa. El feminismo y la subversión de la identidad. México: Paidos.

Butler, J. (2002) Cuerpos que importan. Sobre los límites materiales y discursivos del "sexo". Buenos Aires: Paidós.

Caldas-Coulhtdar, C. y Van Leeuwen, T. (2004) Discurso crítico e gênero no mundo infantil: brinquedos e a representação de atores sociais. Linguagem em (Dis)curso - LemD, Tubarão, v. 4, n.esp, p. 11-33.

Coller, X. (2000) Cuadernos metodológicos. Estudios de Caso. Madrid: Centro de Investigaciones Sociológicas.

Corbetta, P. (2007). Metodología y técnicas de la investigación social. México: Mc Graw Hill.

Delgado, J. M. y Gutiérrez, J. (Coord.) (1999) Métodos y técnicas cualitativas de investigación en Ciencias Sociales. Madrid: Síntesis.

Fernández, A. (1994) La invención de la niña. Buenos Aires: UNICEF.

Mc. Phail Fanger, E. (2006) Voy atropellando tiempos: género y tiempo libre. México: UAM.

Preciado, P. B. (2002). Manifiesto Contrasexual. Prácticas subversivas de identidad sexual. Madrid: Opera Prima.

Reina, M. y Cea, M. (2005) Estereotipos de género en el juego y en el ocio tecnológico interactivo. España: Universidad de Granada, Facultad de Bellas Artes.

Scheines, G. (1999) Juegos inocentes, juegos terribles. Conferencia realizada en la Facultad de Filosofía y Letras, Universidad de Buenos Aires. y organizada por al Área Interdisciplinaria de Estudios del Deporte el 10/4/1999.pp 1-16.

Scott, J. (1986) El género: Una categoría útil para el análisis histórico. En Lamas Marta Compiladora, El género: la construcción cultural de la diferencia sexual. PUEG, México. 265-302 p.

Scott, J. (2008) Preguntas no respondidas. Artículo aparecido en American Historical Review, vol 113, núm. 5, diciembre de 2008, como parte del ahr Forum, Revisiting "Gender: A Useful 
Category of Historical Analysis". Consulta realizada el 8 de marzo de 2015.http://www.debatefeminista.pueg.unam.mx/wpcontent/uploads/2016/03/articulos/040_08.pdf. 